\title{
Psychiatric illness through the lifespan
}

\author{
Frank Schneider • Michael Grözinger
}

Published online: 21 October 2010

(C) Springer-Verlag 2010

Each person can be affected by mental illness, independently of his or her age or gender. Nevertheless, it has been observed that some specific diseases do appear more often in people of a particular age and that reversely the age of the person may also have an influence on the specific characteristics of the psychiatric symptoms. Moreover, the variation of symptoms of a disease through the years indicates that psychiatric illness has always to be seen in relation to the individual background of the individual. One reason for the age-specific modulation of the pathology is related to the influence of biologic and psychosocial factors that change throughout life. As a result, we are only able to comprehend mental disorders of adults if we comprehend the precursors of the illness from the beginnings of cerebral development.

The annual congress of the German Association for Psychiatry and Psychotherapy (Deutsche Gesellschaft für Psychiatrie, Psychotherapie und Nervenheilkunde, hence DGPPN) held in Berlin in November 2009 especially addressed the theme "Psychiatric illness through the lifespan". With this supplement, we intend to give you an overview about the major topics. We have therefore collected a series of invited contributions based on the presentations given during the Berlin congress of Psychiatry to emphasize some aspects of psychiatric illness through one's life. Important questions, especially with regard to the ongoing demographic development in our society were addressed. A number of articles in this supplement refer to this focus: Does anticholinergic drug use enhances the risk

F. Schneider $(\bowtie) \cdot$ M. Grözinger Department of Psychiatry and Psychotherapy, RWTH Aachen University, Pauwelsstr. 30, 52074 Aachen, Germany

e-mail: fschneider@ukaachen.de of dementia in older ages? How do traumatic or lifethreatening experiences influence our brains and lives? How does preventive intervention in persons at risk for psychotic disorders look like?

The diagnosis and therapy of psychiatric illness with regard to the individual situation of the patient was one of the key topics of the Berlin congress of Psychiatry. Furthermore, the prevention of psychiatric diseases, the examination of psychosocial and biologic influences on mental illness, as well as advances in psycho- and pharmacotherapy, were topics that were focused on. We therefore also selected some more clinical articles for this supplement, reflecting actual developments, e.g. concerning therapeutic interventions in patients with affective disorders, individualized treatment of alcohol-dependent patients as well as advances of pharmacotherapy in the treatment of anxiety disorders.

A special focus on our congress was also put on ethics in psychiatry, especially concerning psychiatric research. Consequently, we included a number of articles related to ethical guidelines in psychiatric research, e.g. one article dealing with conflict of interests. Another manuscript focuses on a historical perspective in ethics - the role of ethics in psychiatry in Nazi-Germany. The historical perspective and the coming to terms with the past have become an important focus of the German Association. We are convinced that the role of psychiatry and the psychiatric association in times of Nazi-Germany have not been addressed intensively up to now.

This supplement comprises the major topics of the last congress; nevertheless, it is confined due to the space limits and it reflects a subjective selection. We are sorry that a lot of topics could not be included in this supplement. We hope that our selection of articles will contribute to the discussion about "Psychiatric illness through the lifespan" 
and we look forward to the annual DGPPN congress 2010 that has the main theme "Interdisciplinary Psychiatry".

F. Schneider, M.D., Ph.D.

President of the German Association for Psychiatry and Psychotherapy.

\section{Grözinger, M.D.}

Secretary to the Board of the German Association for Psychiatry and Psychotherapy. 